\title{
Identification of prognostic biomarkers of prostate cancer with long non-coding RNA-mediated competitive endogenous RNA network
}

\author{
GUOMEI YE, LIQIANG GUO, YANFEI XING, WENDONG SUN and MINGZHEN YUAN
}

Department of Urology, The Second Hospital of Shandong University, Jinan, Shandong 250033, P.R. China

Received May 31, 2018; Accepted February 5, 2019

DOI: $10.3892 / \mathrm{etm} .2019 .7277$

\begin{abstract}
Prostate cancer (PCa) is the second most frequently diagnosed male cancer, and no treatments exist for effective inhibition of metastatic spread of PCa. Long non-coding RNA (lncRNA) plays key roles in pathogenesis and development of various cancers through competing with endogenous RNAs (ceRNAs), but at present research on lncRNA functions in PCa is still very limited. Hence, this aspect was investigated using bioinformatics methods. Firstly, the functional lncRNA-mediated ceRNA network associated with PCa was constructed by the multi-step computational approach. Then the cytoscape software was used to analyze the node degree and betweenness centrality (BC) value of lncRNAs and mRNAs in the interaction. Finally, the lncRNAs were screened in the central region of the network by the node degree and $\mathrm{BC}$ value, and the functional enrichment of mRNAs was evaluated with the Gene Ontology (GO) database. In our results, LINC00476, MALAT1, SNHG11, LINC00649, and ILF3-AS1 are the lncRNAs which have the most nodes and higher $\mathrm{BC}$ values and considered as prognostic markers in PCa. GO analysis suggested that the function of screened IncRNAs was obviously focused on intracellular receptor signaling pathway, which indicated these IncRNAs might be potential biomarkers for diagnosis, evaluation and gene-targeted therapy of PCa.
\end{abstract}

\section{Introduction}

Prostate cancer (PCa) is a common cause of cancer-related mortality worldwide, and it is the second most frequently diagnosed male cancer (1). Androgen-deprivation therapy (ADT) has been used as the first-line treatment for PCa, and more than $90 \%$ of PCa patients after surgical removal can be

Correspondence to: Dr Mingzhen Yuan, Department of Urology, The Second Hospital of Shandong University, 247 Beiyuan Street, Jinan, Shandong 250033, P.R. China

E-mail: yuan_mingzhen@126.com

Key words: lncRNA, mRNA, ceRNA network, topology, prostate cancer alleviated (2). Previous studies have shown that the reasons of death in the PCa patients actually results from the dissemination of cancer cells and the establishment of metastases in lymph nodes or bones, but at present no treatments can inhibit the metastatic spread of PCa effectively. Although the conventional histological diagnosis can provide valuable information for treatment, it is not sufficient to predict clinical outcomes (3). Therefore, the appropriate molecular biomarkers are urgently required to diagnose $\mathrm{PCa}$ patients and predict their prognosis.

Due to the development of high-throughput sequencing technology, abundant long non-coding RNAs (lncRNAs) have been discovered in extensive biological processes and attracted wide attention in the medical community. The lncRNAs belong to non-protein-coding RNAs with the length of more than 200 nucleotides. Scholars have suggested that plenty of lncRNAs play complex and critical roles in the progression and pathology of tumors. Terracciano et al considered that the IncRNA overexpression in human cancer cells could distinguish among distinct cancer types unambiguously and could be used as prognostic factors in various cancers (4). In endometrial cancer, a comparison of lncRNAs between different patients identified the lncRNAs with differential expression (5). After functional analysis of these lncRNAs, 5 lncRNAs were found to play key roles in the development of cancer through regulating important related biological processes. This is sufficient to prove that lncRNAs can be regarded as the molecular biomarkers of cancer for diagnosis or prognosis prediction.

The lncRNA functions of PCa are not well characterized, thus the precise identification of IncRNA biomarkers is difficult (6). The studies have corroborated that 1 ncRNAs regulate expression of other transcripts to compete with endogenous RNAs (ceRNAs) $(7,8)$. Recent studies have confirmed that the ceRNA network was associated with lncRNAs in many different cancers, and recognized the prognostic lncRNAs by network analysis $(9,10)$. Some scholars have developed several ceRNA-related databases to facilitate interference of lncRNA function. The databases uncovered the significance of ceRNAs interactions with IncRNA, and declared that the synthesis of network analysis and expression profiles could recognize the risk lncRNAs and the underlying pathology of cancer. Therefore, the functional 1ncRNA-mediated ceRNA network (LMCN) associated with PCa was constructed by the multi-step computational approach in this investigation. 
This study applied a multi-step computational approach to construct a functional $\mathrm{LMCN}$ associated with CRC, and the relevant IncRNAs were identified based on the constructed landscape map. Then functional enrichment analyses were carried out for mRNAs which were significantly associated with lncRNAs, and used the functions of the mature mRNAs to forecast the IncRNA functions.

\section{Materials and methods}

The expression profiles of IncRNA and $m R N A$ in PCa. The gene expression profile was obtained from the Gene Expression Omnibus (GEO) database (http://www.ncbi.nlm. nih.gov/geo/). The dataset is no. GSE72220 which was deposited by Elai and Davicioni based on GPL5175. It has a total of 147 data in the dataset, of which 90 samples are control group and 57 samples are the tumor group. The information of dataset was changed into expressionSet by computer, and stored to the working directory. The expressionSet was preprocessed by the software after finding the relevant working directory in the software analysis page, and a total of 14,451 genes were received.

Clinical characteristics of samples in the profiles. The dataset consisted of 90 normal tissues and 57 prostate tumor tissues. The characteristic of all the samples is RNA. The first step of sampling is dissecting a part of the tumor or normal tissue in the corresponding area of the slide with a sterile biopsy punch tool to extract RNAs. Then the biopsy was scraped from the slide for nucleic acid extraction. Finally, the extraction and purification of total RNA used the RNeasy FFPE kit (Qiagen, Inc., Valencia, CA, USA), and the amplification and marking of RNA used the Ovation WTA FFPE system (Nugen Technologies, Inc., San Carlos, CA, USA).

Clarification of the interaction relationship between IncRNA, $m i R N A$ and $m R N A$. The LMCN was constructed by integrating the expression profiles between IncRNA and mRNA and analyzing the interactions of miRNA-target. The first step is to download miRNA-mRNA interactions and lncRNA-miRNA pinsections from starBase v2.0. The starBase 2.0 is a network which decodes miRNA-ceRNA, miRNA-lncRNA and protein-RNA interactions from 108 sets of CLIP-Seq data generated from 37 independent studies. In the second step, the overlap on the mRNA and lncRNA between the expression profile and the interactions of miRNA-mRNA and IncRNAmiRNA were obtained. A new expression profile was obtained which had a total of 8,522 genes including 8,471 mRNAs and 51 lncRNAs. Finally, new interactions were collected from aforesaid miRNA-mRNA interactions and lncRNA-miRNA intersections which had 265,782 pairs of miRNA-mRNA interactions and 598 pairs of lncRNA-miRNA.

Identification of ceRNA interactions. To evaluate the statistical significance of the shared miRNAs between each lncRNA and mRNA, the hypergeometric test was used for identifying competing lncRNA-mRNA interactions. Because the hypergeometric test assessment and enrichment of miRNAs have interactions of lncRNA and mRNA could achieve this goal. The formula of calculation is as follows (formula 1).

$$
P=1-\sum_{t=0}^{x} \frac{\left(\begin{array}{l}
K \\
t
\end{array}\right)\left(\begin{array}{l}
N-K \\
M-t
\end{array}\right)}{\left(\begin{array}{l}
N \\
M
\end{array}\right)}
$$

In formula $1, \mathrm{~N}$ is the total number of miRNAs, of which $\mathrm{K}$ and $\mathrm{M}$ are the numbers of miRNAs connected with the current lncRNA and mRNA, and $x$ is the number of common miRNA shared by the IncRNA and mRNA. The calculated P-value is used to evaluate the enrichment of the function. It is noteworthy that the false discovery rate (FDR) is used to correct the P-value and the FDR $<0.01$ regarded as the threshold. After correction, the LMCN with P-value $<0.01$ contained 51 lncRNAs, 8,125 mRNAs and 34,586 interactions.

Screening the subnetworks of LMCN (sub-LMCN) regulated by the highly competitive lncRNA. To analyze the co-expression of the screened lncRNA-mRNA interactions, Pearson's correlation coefficients of the control and disease groups were calculated. The formula of calculation is as follows (formula 2).

$$
\rho_{X, Y}=\frac{\operatorname{cov}(X, Y)}{\sigma_{X} \sigma_{Y}},
$$

In formula 2, $\operatorname{cov}(X, Y)$ is the covariance of variables $X$ and $Y$, of which $\sigma_{X}$ and $\sigma_{Y}$ are the standard deviations for $X$ and $Y$, respectively. FDR $<0.01$ was regarded as the threshold. The difference value of Pearson's correlation coefficient was $>0.45$ and considered as the significant coexpression of the ceRNA interactions.

Prediction of IncRNA function. The prediction of lncRNA function used the mature function of mRNA though functional enrichment analysis of the mRNAs which were significantly correlated with lncRNA. First of all, the Gene Ontology (GO) analysis was used for prediction. Using the $\mathrm{P}=0.01$ as the threshold, the enrichment analysis of mRNAs in the subLMCN was based on the BP classification of GO analysis. The pathway was subsequently predicted. In this study, the pathway database was KEGG, and the Fisher test was used to identify the enrichment pathway of lncRNA. After eliminating the pathway with enrichment $\mathrm{P}$-value $<0.05$, the other results were the pathway which may be regulated by lncRNA.

\section{Results}

Structure of LMCN. This study constructed an LMCN to assess the 1ncRNA-mediated ceRNA interaction landscape. A multi-step approach was used to accomplish this. lncRNA transcripts that compete with endogenous mRNAs to bind miRNAs could be identified by the present miRNA target prediction methods. To identify the interactions with miRNA-lncRNA and miRNA-mRNA of PCa, starBase v2.0 was applied to decode and collect data sets in largescale CLIP-seq data. Unlike the results predicted by current software, the data set obtained from starBase v2.0 provided high confidence miRNA-target interactions supported by the experiments. The new expression profiles of 8,522 genes were obtained, which contained 8,471 mRNAs and 51 lncRNAs after assessment of intersection between the obtained data set and the genes of the chip platform HuEx-1_0-st (Affymetrix 

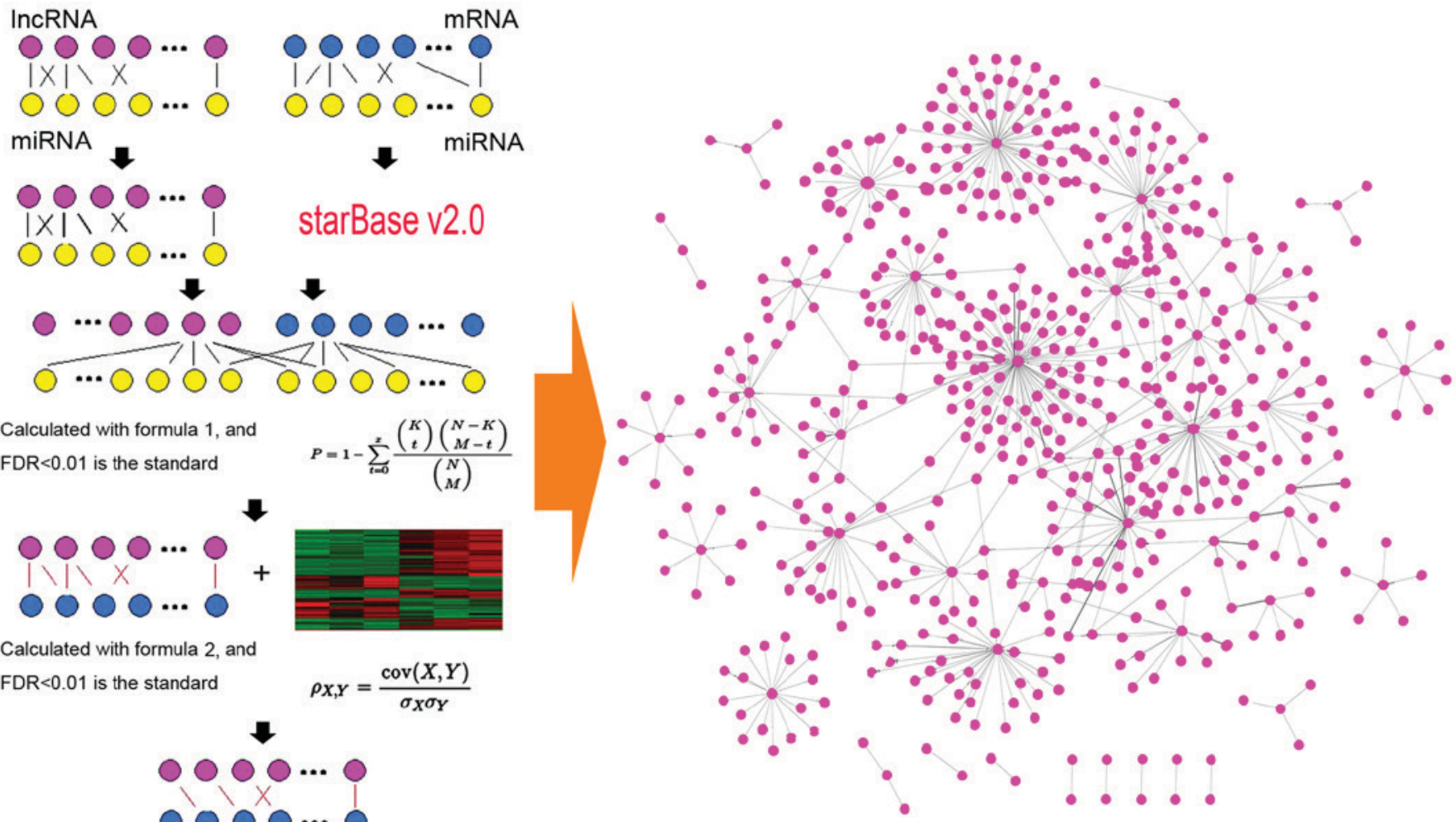

Figure 1. The flow chart of LMCN construction. The left panel shows the construction process of LMCN. The right panel is the preliminary sketch of LMCN. LMCN, lncRNA-mediated ceRNA network; lncRNA, long non-coding RNA.

Human Exon 1.0 ST Array), as well as and combining mRNA and lncRNA in the data set. In this new expression profile, there were 265,782 pairs of miRNA-mRNA interactions and 598 pairs of IncRNA-miRNA intersections. Then the LMCN model was constructed with the significantly co-expressed lncRNA-mRNA ceRNA pairs (Fig. 1).

Biological and topological properties revealed by LMCN. The hypergeometric test was used to evaluate the enrichment of miRNAs, and 34,586 pairs of interactions which included 51 lncRNAs and 8,125 mRNAs after screened by FDR correction were obtained. The statistics of nodes degree were obtained to reveal power law distribution, and the $\mathrm{R}^{2}=0.999$ is the PCa-associated LMCN, a scale-free network (Fig. 2). Then the node degree and betweenness centrality (BC) of LMCN were analyzed, which are topological attributes. The box plots (Fig. 3A and B) show the statistics of the degree and $\mathrm{BC}$ value which included the maximum value, quartile value and median value. The scatter plots (Fig. 3C and D) show the distribution of IncRNAs and mRNAs. A higher degree demonstrated that the node was a hub, which was involved in more ceRNA interactions. A higher BC showed that the node was a bottleneck, which acted as bridges connecting different network modules. Compared to mRNA nodes, the lncRNA nodes show more specific topological properties because of their greater degrees and $\mathrm{BC}$ values.

Critical lncRNAs of PCa. The Pearson correlation coefficient of the screened lncRNA-mRNA interactions was calculated between the control and PCa groups using co-expression analysis. The difference value (D-value) of Pearson correlation

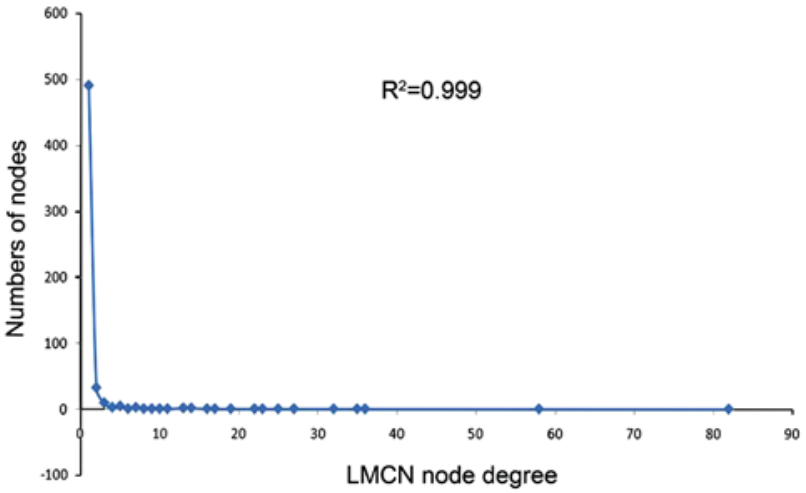

Figure 2. The power-law distribution of LMCN node degree. The dots are the node number of 1 ncRNAs and mRNAs in LMCN. $\mathrm{R}^{2}$ value shows the fitting degree of the curve, and the value closer to 1 , has the better fitting degree. LMCN, lncRNA-mediated ceRNA network; lncRNA, long non-coding RNA.

coefficient was calculated for the same interaction between the control and PCa groups, and the interactions with D-value $>0.45$ were regarded as the significant coexpression ceRNA interactions. After mapping the network graph, it was found that there were 46 lncRNAs, 522 mRNAs and 569 interactions (Fig. 4). In addition, the results showed that the lncRNA nodes were always in the central region of the network, whereas the mRNA nodes were always in the outer layer. Therefore, in the LMCN of PCa, the IncRNAs dominated in the central regulatory function. Using the cytoscape software to analyze the ceRNA interactions, it was found that LINC00476, MALAT1, SNHG11, LINC00649, and ILF3-AS1 had more nodes and higher BC compared to other lncRNAs. 

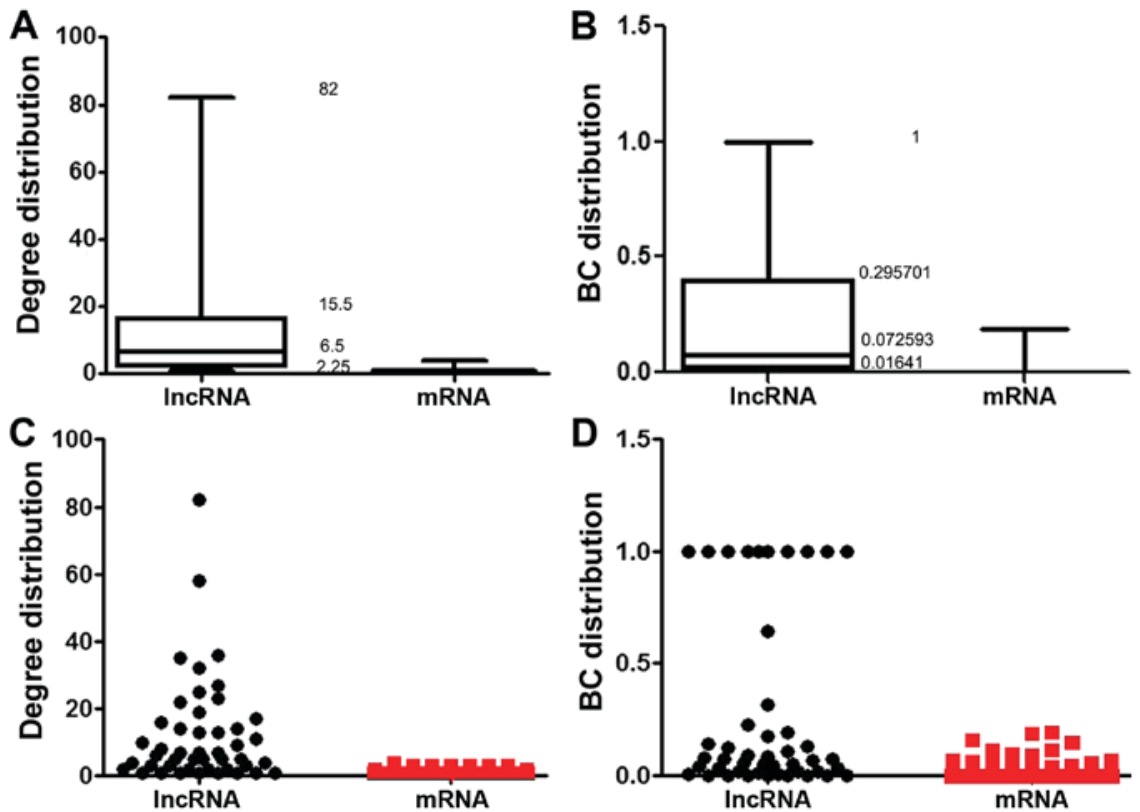

Figure 3. (A and B) The box plots of node degree and $\mathrm{BC}$ value distribution. (C and D) The scatter plots of node degree and $\mathrm{BC}$ value distribution. In the box plots, the 4 values of the lines, respectively, represent the maximum value, the first quartile, the median value, and the third quartile. BC, betweenness centrality.
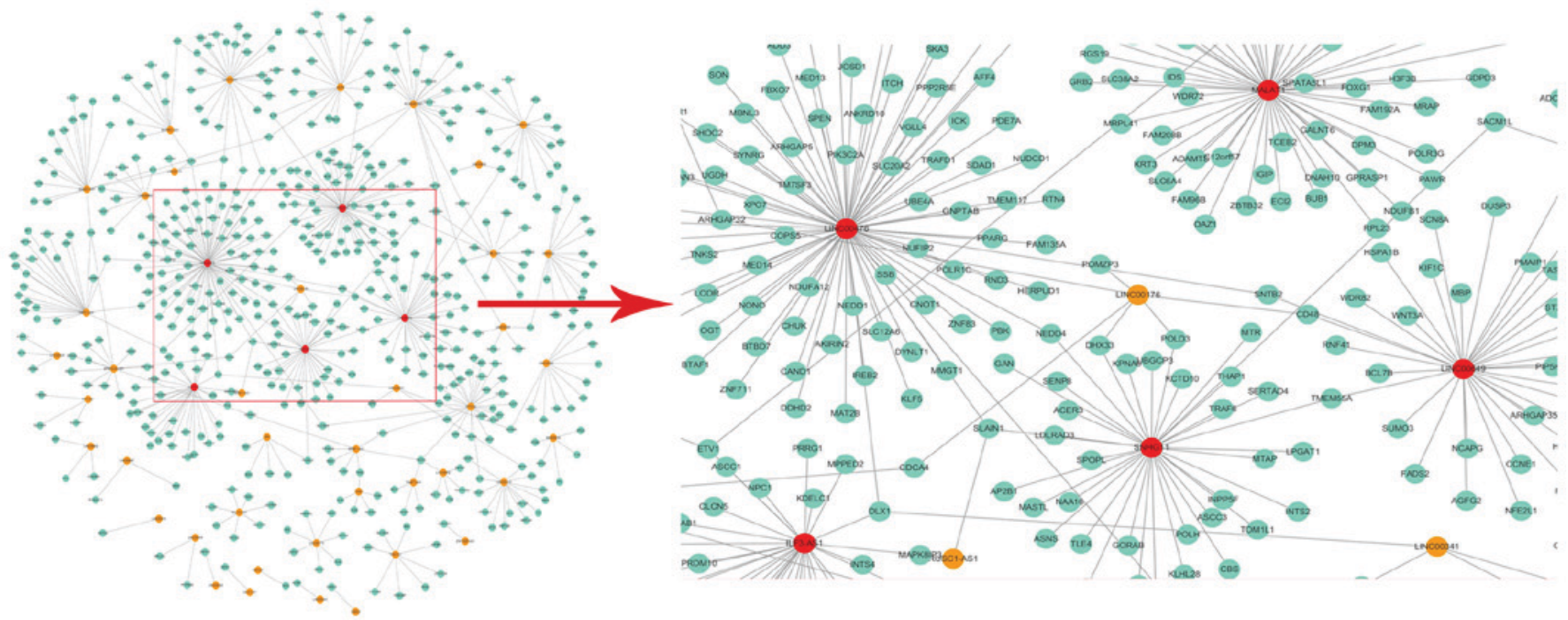

Figure 4. On the left is the cytoscape of the interactions. On the right are the lncRNAs which were chosen as the prognostic markers in PCa. The green spots are the mRNAs; the orange spots are the lncRNAs; the red spots are the lncRNAs with the most nodes and higher BC values. IncRNA, long non-coding RNA; $\mathrm{PCa}$, prostate cancer;BC, betweenness centrality.

It suggests that these 5 lncRNAs are likely to be prognostic markers in $\mathrm{PCa}$.

Prediction of GO analysis and pathway. In order to further verify the potential function of lncRNAs, the functional enrichment of mRNAs was analyzed, which were significantly associated with lncRNA and the function of lncRNA can be predicted by the mature function of mRNA. The mRNAs which are closely related to the central lncRNAs of PCa have an obvious enrichment in a function whose ID is GO: 0030522, and the involved genes are DAB2, ASXL1, CTNNB1, TRIM16, SAFB, DDRGK1, VDR, EZH2, TWIST1, CCNE1, HSPA1B, PUM1, NOTCH2, TRAF6, NEDD4, MED13, ITCH, PPARG, $M E D 14, C H U K, C N O T 1, B R D 8$, and SRC. The pathway of the
lncRNA enrichment was identified by the Fisher Test based on the Kyoto Encyclopedia of Genes and Genomes (KEGG) database. After correction, the pathway with P-value $<0.05$ was the pathway for enrichment of mRNAs that was significantly associated with IncRNA, indicating they may be the pathway regulated by the IncRNAs (Table I).

\section{Discussion}

The early treatments of PCa include surgical treatment, androgen blockade and radiotherapy, but most patients still suffered from recurred tumors which will destroy the bone marrow and lead to death (11). Therefore, it is urgent to identify the molecular mechanisms to confirm potential 
Table I. The prediction of GO analysis and pathways.

\begin{tabular}{llc}
\hline Pathway & \multicolumn{1}{c}{ P-value (FDR) } & Number of mRNA \\
\hline 4144 Endocytosis [PATH:hsa04144] & 0.0178827965638394 & 18 \\
4510 Focal adhesion [PATH:hsa04510] & 0.020522962511304 & 16 \\
4740 Olfactory transduction [PATH:hsa04740] & 0.020522962511304 & 2 \\
5200 Pathways in cancer [PATH:hsa05200] & 0.0196461610273246 & 26 \\
5222 Small cell lung cancer [PATH:hsa05222] & 0.0196461610273246 & 10 \\
5169 Epstein-Barr virus infection [PATH:hsa05169] & 0.020522962511304 & 16
\end{tabular}

GO, Gene Ontology; FDR, false discovery rate.

effective therapeutic targets for improving patient survival. Bioinformatics is one of the interdisciplinary disciplines involved in many fields, and one of its core elements was to study the internal expression regulatory mechanisms of genes and to reveal essential laws of human diseases (12). IncRNAs are longer than 200 nucleotides but the functional open reading frames are lackig. IncRNAs have important clinical value of diagnosis, prognosis and treatment because of their biological properties, so that it has been extensively studied. The studies indicated that lncRNAs participate in a variety of cell biological processes such as cellular proliferation, differentiation, and DNA damage responses (13). At present, the aberrant expression of lncRNAs occurs in many human diseases. Aiello et al using RNA-ChIP and ChIRP found that in PCa patients HOTAIR and MALAT1 were relevant to both ER $\alpha / E R \beta$ detectable at chromatin level (14). The results showed that MALAT1 silencing was relevant for PCa, and disclosing potential perspective manipulations in terms of transcription regulation of prognostic genes.

It has been identified that lncRNA overexpression is concerned with the progression and pathology of PCa (15). The PCa-specific LncRNAs affected PCa cell self-renewal, proliferation, survival, metastasis, and apoptosis by either transcriptional or post-transcriptional regulation. Therefore some of them are involved in distinct subtypes of PCa (16). To make clear the intermodulation relationship between lncRNA and mRNA, a functional LMCN was constructed. First the gene expression profile collected in GEO database was preprocessed, resulting in obtaining 14,451 genes. Then the miRNA-mRNA interactions and lncRNA-miRNA pinsections from starBase v2.0, were studied and new gene expression profile and interaction pairs though overlapping miRNAmRNA and lncRNA-miRNA interaction were obtained. The new gene expression profile included 8,522 genes, and the new interaction pairs included 265,782 pairs of miRNA-mRNA interactions and 598 pairs of lncRNA-miRNA. Finally, in order to assess the significance of miRNAs shared between each mRNA and lncRNA, competitive lncRNA-mRNA interactions were identified using the hypergeometric test, and the prediction of lncRNA function used the mature mRNA function.

The lncRNAs are implicated in regulation of PCa generation and development, and their functions are accomplished by adjusting the expression level of protein-coding genes. In this study, the interaction between IncRNA and mRNA was analyzed using software, and most of the central points were lncRNA. The power law distributions shown as $\mathrm{R}^{2}=0.999$ indicated the LMCN network has a high degree of confidence. Compared the number of nodes and $\mathrm{BC}$ values among different central lncRNAs, LINC00476, MALAT1, SNHG11, LINC00649 and ILF3-AS1 had the deeper interaction with more mRNAs, therefore these 5 lncRNAs were more likely to serve as the prognostic markers of PCa. MALAT1 is also named NEAT2, and was a highly conserved lncRNA. Studies have shown that it was overexpressed in multifarious human tumors and linked to enhanced cell migration, invasion and proliferation of cancers. The human tumors affected by MALAT1 included glioma (17), pancreatic (18), prostate (19) and lung cancer (20). Sebastian et al thought that the cancer metastasis was a complex process in which the cells deviated from the cancer primary site then traveled through the lymphatic or circulatory systems to become secondary tumors (21). They used PCa-osteoblast interaction microarrays to recognize the new factors which promote PCa metastasis to bone. In the result, they found that MALAT1 was not only one of the differentially expressed genes, but also regulated by Sost. It meant that the proliferation, migration, or invasion of PCa cells may be regulated by the change of MALAT1 expression. Unlike MALAT1, the other four lncRNAs had no verified experiments or studies in connection with PCa. However, it was discovered that the expression of ILF3AS1 is negatively correlated with that of miR-200b/a/429 in melanoma tissues (22). ILF3-AS1 is a melanoma-upregulated lncRNA induces cell invasion, migration and proliferation through negatively regulating miR-200b/a/429. Chaudhry investigateded the miRNA modulation mechanism in human cells treated by radiotherapy (23). SNHG11 was induced in TK6 and WTK1 cells, and its expression level was followed by a decline. SNHG11 has been well-characterized in cancer. Although the direct study between these four lncRNAs and PCa was lacking, their effects on various cancers were unquestionable. Therefore, the potential lncRNA biomarkers (e.g. LINC00476, MALAT1, SNHG11, LINC00649 and ILF3AS1) have been inferred, which can be used for diagnosis, evaluation and gene-targeted therapy of PCa. Further studies need to be carried out for verifying this inference. The GO analysis suggested that the occurrence and progression of $\mathrm{PCa}$ were associated with intracellular receptor signaling pathway. The Fisher test indicated that IncRNAs may be involved in Endocytosis and Focal adhesion. 
In conclusion, this study structured an LMCN landscape of 147 PCa samples with a new method. The LMCN landscape was used to observe the specific topological properties and synergistic, competitive effects of lncRNAs, and it revealed regulatory interactions with coding mRNAs in PCa.

\section{Acknowledgements}

Not applicable.

\section{Funding}

This study is supported by the Natural Science Foundation of Shandong (grant no. ZR201709290020).

\section{Availability of data and materials}

The datasets used and/or analyzed during the present study are available from the corresponding author on reasonable request.

\section{Authors' contributions}

GY and LG conceived the study and drafted the manuscript. YX acquired the data. WS and MY analyzed the data and revised the manuscript. All authors read and approved the final manuscript.

\section{Ethics approval and consent to participate}

Not applicable.

\section{Patient consent for publication}

Not applicable.

\section{Competing interests}

The authors declare that they have no competing interests.

\section{References}

1. Kalsbeek AMF, Chan EKF, Corcoran NM, Hovens CM and Hayes VM: Mitochondrial genome variation and prostate cancer: a review of the mutational landscape and application to clinical management. Oncotarget 8: 71342-71357, 2017.

2. Lee SU and Cho KH: Multimodal therapy for locally advanced prostate cancer: The roles of radiotherapy, androgen deprivation therapy, and their combination. Radiat Oncol J 35: 189-197, 2017.

3. Puche-Sanz I, Alvarez-Cubero MJ, Pascual-Geler M, Rodriguez-Martinez A, Delgado-Rodriguez M, Garcia-Puche JL, Expósito J,Robles-Fernández I,Entrala-Bernal C,Lorente JA, et al: A comprehensive study of circulating tumour cells at the moment of prostate cancer diagnosis: Biological and clinical implications of EGFR, AR and SNPs. Oncotarget 8: 70472-70480, 2017.

4. Terracciano D, Terreri S, de Nigris F, Costa V, Calin GA and Cimmino A: The role of a new class of long noncoding RNAs transcribed from ultraconserved regions in cancer. Biochim Biophys Acta 1868: 449-455, 2017.

5. Sun Y, Zou X, He J and Mao Y: Identification of long non-coding RNAs biomarkers associated with progression of endometrial carcinoma and patient outcomes. Oncotarget 8: 52604-52613, 2017.

6. Su W, Xu M, Chen X, Chen N, Gong J, Nie L,Li L, Li X, Zhang M and Zhou Q: Long noncoding RNA ZEB1-AS1 epigenetically regulates the expressions of ZEB1 and downstream molecules in prostate cancer. Mol Cancer 16: 142, 2017.
7. Jia J, Li F, Tang XS, Xu S, Gao Y, Shi Q, Guo W, Wang X, He D and Guo P: Long noncoding RNA DANCR promotes invasion of prostate cancer through epigenetically silencing expression of TIMP2/3. Oncotarget 7: 37868-37881, 2016.

8. Misawa A, Takayama K, Urano T and Inoue S: Androgen-induced long noncoding RNA (lncRNA) SOCS2-AS1 promotes cell growth and inhibits apoptosis in prostate cancer cells. J Biol Chem 291: 17861-17880, 2016.

9. Wu H, Wu R, Chen M, Li D, Dai J, Zhang Y, Gao K, Yu J, Hu G, Guo Y, et al: Comprehensive analysis of differentially expressed profiles of lncRNAs and construction of miR-133b mediated ceRNA network in colorectal cancer. Oncotarget 8: 21095-21105, 2017.

10. Zhou M, Diao Z, Yue X, Chen Y, Zhao H, Cheng L and Sun J: Construction and analysis of dysregulated lncRNA-associated ceRNA network identified novel lncRNA biomarkers for early diagnosis of human pancreatic cancer. Oncotarget 7: 56383-56394, 2016.

11. Makishima H, Ishikawa H, Tanaka K, Mori Y, Mizumoto M, Ohnishi K, Aihara T, Fukumitsu N, Okumura T and Sakurai H: A retrospective study of late adverse events in proton beam therapy for prostate cancer. Mol Clin Oncol 7: 547-552, 2017.

12. Ready D, Yagiz K, Amin P, Yildiz Y, Funari V, Bozdag S and Cinar B: Mapping the STK4/Hippo signaling network in prostate cancer cell. PLoS One 12: e0184590, 2017.

13. Wu Y, Liang S, Xu B, Zhang R, Zhu M, Zhou W, Zhang S, Guo J, $\mathrm{Xu} \mathrm{L}$ and Zhu $\mathrm{H}$ : Long noncoding RNA eosinophil granule ontogeny transcript inhibits cell proliferation and migration and promotes cell apoptosis in human glioma. Exp Ther Med 14: 3817-3823, 2017.

14. Aiello A, Bacci L, Re A, Ripoli C, Pierconti F, Pinto F, Masetti R, Grassi C, Gaetano C, Bassi PF, et al: MALAT1 and HOTAIR long non-coding RNAs play opposite role in estrogen-mediated transcriptional regulation in prostate cancer cells. Sci Rep 6: 38414, 2016.

15. Ahadi A, Brennan S, Kennedy PJ, Hutvagner G and Tran N: Long non-coding RNAs harboring miRNA seed regions are enriched in prostate cancer exosomes. Sci Rep 6: 24933, 2016.

16. Mehra R, Udager AM, Ahearn TU, Cao X, Feng FY, Loda M, Petimar JS, Kantoff P, Mucci LA and Chinnaiyan AM: Overexpression of the long non-coding RNA SChLAP1 independently predicts lethal prostate cancer. Eur Urol 70: 549-552, 2016.

17. Cao S, Wang Y, Li J, Lv M, Niu H and Tian Y: Tumor-suppressive function of long noncoding RNA MALAT1 in glioma cells by suppressing miR-155 expression and activating FBXW7 function. Am J Cancer Res 6: 2561-2574, 2016.

18. Jiao F, Hu H, Yuan C, Wang L, Jiang W, Jin Z, Guo Z and Wang L: Elevated expression level of long noncoding RNA MALAT-1 facilitates cell growth, migration and invasion in pancreatic cancer. Oncol Rep 32: 2485-2492, 2014.

19. Ren S, Liu Y, Xu W, Sun Y, Lu J, Wang F, Wei M, Shen J, Hou J, Gao X, et al: Long noncoding RNA MALAT-1 is a new potential therapeutic target for castration resistant prostate cancer. J Urol 190: 2278-2287, 2013.

20. Tano K, Mizuno R, Okada T, Rakwal R, Shibato J, Masuo Y, Ijiri K and Akimitsu N: MALAT-1 enhances cell motility of lung adenocarcinoma cells by influencing the expression of motility-related genes. FEBS Lett 584: 4575-4580, 2010.

21. Sebastian A, Hum NR, Hudson BD and Loots GG: Cancer-osteoblast interaction reduces sost expression in osteoblasts and up-regulates lncRNA MALAT1 in prostate cancer. Microarrays (Basel) 4: 503-519, 2015.

22. Chen X, Liu S, Zhao X, Ma X, Gao G, Yu L, Yan D, Dong H and Sun W: Long noncoding RNA ILF3-AS1 promotes cell proliferation, migration, and invasion via negatively regulating miR-200b/a/429 in melanoma. Biosci Rep 37: pii: BSR20171031, 2017.

23. Chaudhry MA: Expression pattern of small nucleolar RNA host genes and long non-coding RNA in X-rays-treated lymphoblastoid cells. Int J Mol Sci 14: 9099-9110, 2013.

This work is licensed under a Creative Commons Attribution-NonCommercial-NoDerivatives 4.0 International (CC BY-NC-ND 4.0) License. 